\title{
Productividad del sábalo (Prochilodus lineatus) cultivado en estanques con diferentes tipos de fondo
}

\author{
Della Rosa, P.; Roux, J.P.; Sánchez, S.; Ortiz, J.C.; Domitrovic, H.A. \\ Instituto de Ictiología del Nordeste, Facultad de Ciencias Veterinarias, UNNE, \\ Sargento Cabral 2139, Corrientes (3400), Argentina. E-mail: paodr88@live.com
}

\begin{abstract}
Resumen
Della Rosa, P.; Roux, J.P.; Sánchez, S.; Ortiz, J.C.; Domitrovic, H.A.: Productividad del sábalo (Prochilodus lineatus) cultivado en estanques con diferentes tipos de fondo. Rev. vet. 25: 2, 126-130, 2014. El sábalo (Prochilodus lineatus) es la especie más importante para la pesca comercial y de subsistencia en Argentina. Sin embargo, hace algunos años alcanzó una situación de riesgo que motivó que el cultivo en cautiverio sea impulsado por diversos sectores públicos y privados debido al interés en la conservación ambiental. Este pez presenta un rápido crecimiento, elevada fecundidad y rusticidad. El objetivo del presente trabajo fue analizar comparativamente estanques de cultivo de $P$. lineatus con dos tipos de fondo, uno de cemento y otro de tierra, a fin de identificar alguna condición que influya sobre la productividad de la especie. Fueron utilizados 450 juveniles de 60 días y $26 \mathrm{~g}$ de peso vivo promedio, a quienes se suministró alimento balanceado en base al $2 \%$ de la biomasa. Las variables de calidad de agua se registraron periódicamente, al igual que las biometrías, para ajustar la alimentación al crecimiento de los peces. El ensayo tuvo una duración de 340 días. Los parámetros físicoquímicos de calidad de agua permanecieron dentro de valores adecuados para la especie. Las variables productivas analizadas fueron: coeficiente de crecimiento específico, biomasa total (donde se observaron diferencias significativas, $\mathrm{p}<0,05$ ) y tasa de supervivencia (sin diferencias significativas entre ambos tratamientos, $p>0,05$ ). Los resultados obtenidos demuestran que los estanques con fondo de cemento no permiten que el sábalo se desarrolle como lo hace en estanques con fondo de tierra. Sin embargo, representan una alternativa para el cultivo de la especie, que debería continuar evaluándose con el objeto de lograr el mantenimiento de una adecuada cantidad de material orgánico que satisfaga las necesidades de los peces en cultivo.
\end{abstract}

Palabras clave: piscicultura, Prochilodus lineatus, crecimiento, recría, engorde.

\begin{abstract}
Della Rosa, P.; Roux, J.P.; Sánchez, S.; Ortiz, J.C.; Domitrovic, H.A.: Productivity of "sábalo" (Prochilodus lineatus) grown in different types of pond bottoms. Rev. vet. 25: 2, 126-130, 2014. In Argentina, "sábalo" (Prochilodus lineatus) is the most important commercial fishing and subsistence species. However, in the past years the species reached a risk point, which motivated the captive culture driven by various public and private sectors interested in environmental conservation. This fish has a rapid growth, high fertility and rustiness. The aim of this study was to compare culture ponds for P. lineatus with two types of bottoms (concrete vs soil), in order to identify any condition that influence the productivity of the species. Four hundred offspring, 60 days-old and $26 \mathrm{~g}$ of body average weight, fed with balanced food representing $2 \%$ of the biomass, were used. Both water and biometric quality variables were recorded periodically, to adjust feed to fish growth. The experiment lasted 340 days. Water physical and chemical quality parameters remained within appropriate values for the species. Analyzed productive variables were specific growth coefficient, total biomass (with significant differences, $\mathrm{p}<0.05$ ), and survival rate (with no significant differences between treatments, $\mathrm{p}>0.05$ ). Results show that the concrete bottom ponds do not allow "sábalo" development as well as soil ponds do. However, concrete bottom ponds are an alternative for the production of the species which needs further evaluation in order to achieve the maintenance of an adequate amount of organic material that meet the needs of the species.
\end{abstract}

Key words: pisciculture, Prochilodus lineatus, growing, rebreeding, fattening. 


\section{INTRODUCCIÓN}

Los peces del género Prochilodus son los teleósteos detritívoros de mayor extracción en la pesca continental de Sudamérica. En Argentina, el sábalo (Prochilodus lineatus) es la especie más importante para la pesca comercial y de subsistencia ${ }^{14}$. Este pez presenta un alto potencial para el manejo de sus poblaciones naturales, fundamentalmente por su rápido crecimiento, elevada fecundidad y rusticidad, cualidades que posibilitan su adecuada cría en cautiverio ${ }^{4,7}$.

En el litoral fluvial argentino, el sábalo constituye el $75-85 \%$ de las capturas totales anuales en las pesquerías artesanales del río Paraná (medio e inferior) ${ }^{14}$. Este recurso alcanzó una situación de riesgo hace algunos años como consecuencia de la alta presión de pesca acompañada de situaciones climáticas e hidrológicas que determinaron un bajo desempeño de las poblaciones naturales de la especie entre 1998 y $2006^{8}$.

$P$. lineatus es un pez de hábitos migratorios, que fuera del periodo reproductivo frecuenta aguas tranquilas o remansos con fondo blando y fangoso, ambientes que por sus caracteres fisiográficos se asemejan a los del biotipo léntico ${ }^{4,5,22}$. Su bajo nivel trófico dentro de la cadena alimentaria y la aceptación por los consumidores, motivaron su estudio desde hace mucho tiempo con fines productivos. El principal problema para la cría en cautiverio, es la dificultad para encontrar una dieta apropiada que maximice su potencial productivo, constituyéndose en un cuello de botella que impide la expansión de la actividad ${ }^{3,9,18}$, ya que se obtienen bajas tasas de sobrevivencia que dificultan la producción a gran escala, haciéndola muy variable y altamente dependiente de las condiciones ambientales, limitando la proyección de la producción ${ }^{19}$.

Actualmente, iniciativas de diversos sectores públicos y privados, asociadas principalmente al interés en la conservación ambiental, favorecen el desarrollo de la piscicultura, la cual se espera que amplíe los límites de exploración del recurso natural y la obtención de proteína animal de bajo costo ${ }^{20}$. A la fecha, no existe suficiente información referente al cultivo de la especie en estanques con fondo de cemento, técnica que podría permitir una mayor expansión de la actividad si se aprovechan los estanques existentes en numerosas empresas agropecuarias de la región.

En este trabajo se analizan comparativamente estanques de cultivo de P. lineatus con dos tipos de fondo a fin de identificar condiciones que influyan sobre la productividad de la especie.

\section{MATERIAL Y MÉTODOS}

El ensayo se desarrolló en las instalaciones del Instituto Nacional de Tecnología Agropecuaria, Corrientes. Se utilizaron seis estanques de alrededor de $150 \mathrm{~m}^{2}$. Tres de ellos fueron rectangulares, construidos sobre terreno natural, con taludes y fondo de tierra (T0). Los tres restantes fueron del tipo "tanque australiano" con pared de chapa de zinc y fondo de cemento (T1).

En todos ellos se trabajó con una densidad de 0,5 pez $/ \mathrm{m}^{2}$. El ensayo se inició con 450 juveniles de 60 días, con peso vivo promedio de $26 \mathrm{~g}$. Previamente al inicio de la experiencia los estanques fueron fertilizados con estiércol bovino y fardos de alfalfa a razón de $300 \mathrm{~g} / \mathrm{m}^{2}$. Transcurridos siete días de la fertilización se procedió a la medición de variables de calidad de agua para luego asignar aleatoriamente los lotes de peces a cada estanque.

La experiencia tuvo una duración total de 340 días. Diariamente se procedió a medir la temperatura del agua y a suministrar alimento balanceado en base al $2 \%$ de la biomasa total de cada estanque, valor que fue ajustado en cinco oportunidades tras la realización de biometrías parciales llevadas a cabo cada 45 días aproximadamente, para ajustar la alimentación al crecimiento de los peces (no se realizaron biometrías durante el período invernal). En cada biometría se estimó la longitud estándar y el peso a partir de muestras de 25 ejemplares por estanque. Semanalmente y durante todo el periodo experimental, se registraron los datos básicos de calidad de agua como $\mathrm{pH}$, oxígeno disuelto (\% y $\mathrm{mg} / \mathrm{l})$, conductividad, turbidez, nitritos y amoníaco.

Al finalizar el ensayo se vaciaron los estanques y se procedió al recuento y pesaje de todos los peces. Las variables productivas analizadas fueron: tasa de supervivencia $(\mathrm{TS})=\mathrm{N}^{\circ}$ peces final $/ \mathrm{N}^{\circ}$ peces inicial $* 100$; coeficiente de crecimiento especifico $(\mathrm{CCE})=[3$ (peso final ${ }^{0,33}$ - peso inicial $\left.{ }^{0,33}\right) /$ tiempo en días] x 100; y biomasa total $(\mathrm{B})=\mathrm{N}^{\circ}$ peces $\mathrm{x}$ peso medio.

Los datos fueron procesados utilizando el programa InfoStat ${ }^{12}$. Los tratamientos se compararon estadísticamente mediante ANCOVA para cada una de las variables productivas analizadas, considerando a las variables de calidad de agua como covariables. Debido a que ninguna covariable presentó efecto estadísticamente significativo, el modelo se reemplazó por uno más sencillo, realizándose una prueba " $t$ " para muestras independientes. En todas las pruebas estadísticas mencionadas el nivel de significación utilizado fue del $5 \%(p<0,05)$.

\section{RESULTADOS Y DISCUSIÓN}

Los parámetros físico-químicos de calidad de agua permanecieron dentro de valores adecuados para la especie durante todo el periodo experimental (Tabla 1), donde no se observaron diferencias significativas entre los tratamientos ni se vieron afectadas las variables productivas en el ANCOVA ( $p>0,05)$.

Supervivencia. La TS no presentó diferencias significativas entre tratamientos ( $>0,05$; Figura 1), indicando que los peces se adaptaron de manera similar a las condiciones físico-químicas del agua y al manejo en ambos sistemas. Otros investigadores que trabajaron con $P$. lineatus en tanques redes y estanques con fondo de tierra a una densidad de $17 \mathrm{peces} / \mathrm{m}^{3}$ y $1 \mathrm{pez} / \mathrm{m}^{2}$ res- 
Tabla 1. Parámetros de calidad de agua.

\begin{tabular}{lc}
\hline parámetro & promedio $\pm \mathrm{DE}$ \\
\hline temperatura $\left({ }^{\circ} \mathrm{C}\right)$ & $24,79 \pm 0,41$ \\
$\mathrm{pH}(\mathrm{score})$ & $8,57 \pm 0,55$ \\
conductividad $(\mu \mathrm{S} / \mathrm{cm})$ & $286,58 \pm 85,18$ \\
oxígeno $(\mathrm{mg} / \mathrm{l})$ & $10,78 \pm 1,52$ \\
oxígeno $(\%)$ & $122,77 \pm 14,84$ \\
turbidez $(\mathrm{NTU})$ & $5,44 \pm 5,22$ \\
nitritos $(\mathrm{mg} / \mathrm{l})$ & $0,06 \pm 0,05$ \\
alcalinidad $\left(\mathrm{mg} \mathrm{CaCO}_{3} / \mathrm{l}\right)$ & $156 \pm 63,50$ \\
\hline
\end{tabular}

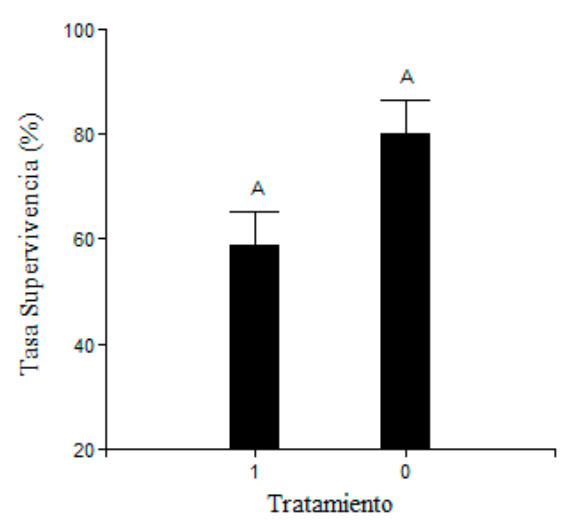

Figura 1. Tasa de supervivencia en estanque con fondo de cemento (T1) y de tierra (T0). Letras iguales indican inexistencia de diferencias significativas.

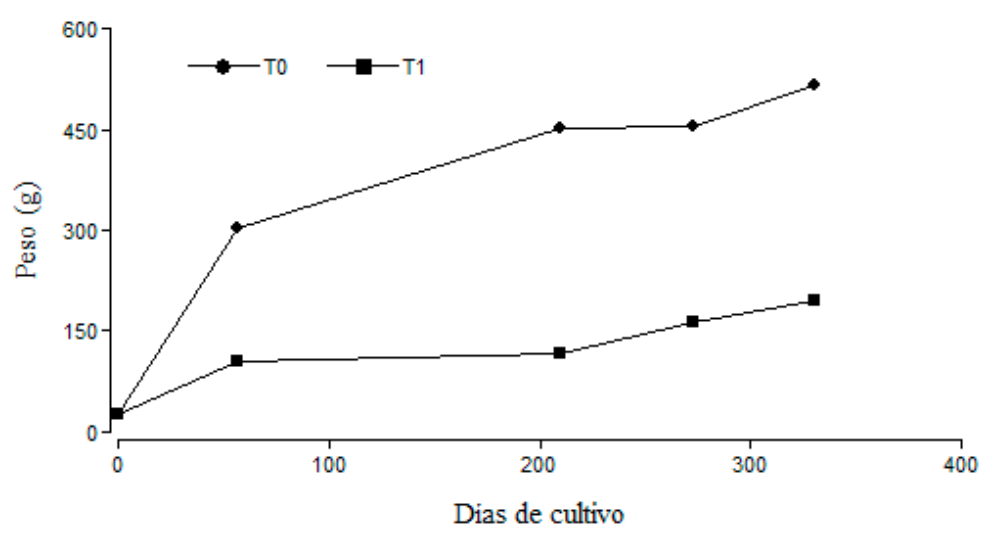

Figura 2. Distribución de pesos de acuerdo a los días de cultivo, en cada tratamiento.
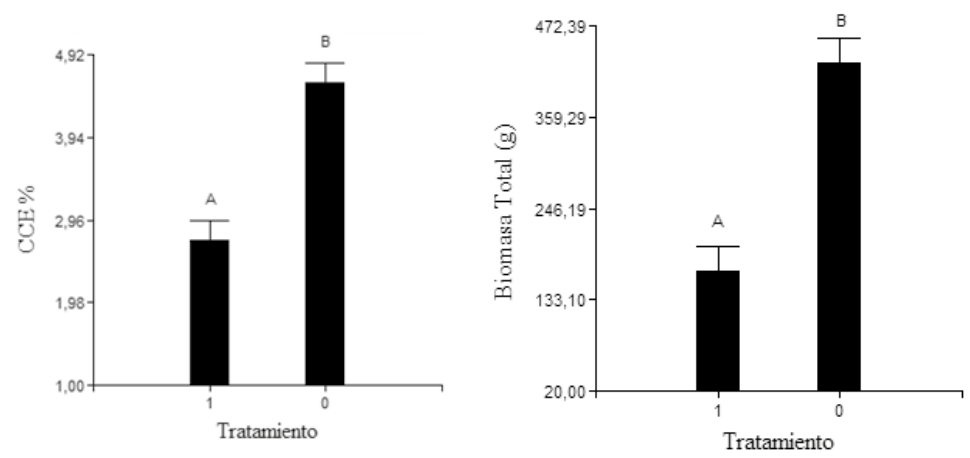

Figura 3. Coeficiente de crecimiento específico (CCE) y biomasa total, según tratamiento. Letras distintas corresponden a diferencias significativas. pectivamente, tampoco registraron diferencias significativas entre tratamientos ${ }^{10}$.

Crecimiento. El peso final fue significativamente diferente entre ambos tratamientos $(\mathrm{p}<0,05$; Figura 2$)$, registrándose pesos superiores en los estanques con fondo de tierra, con promedio de 452,76 $\pm 64,43 \mathrm{~g}$ al final de la experiencia, mientras que en $\mathrm{T} 1$ dicho guarismo fue de 193,87 $\pm 40,83 \mathrm{~g}$. De igual forma, el CCE y la biomasa total producida resultaron superiores en T0 ( $<<0,05$; Figura 3). Estos resultados podrían relacionarse con el aporte de nutrientes al agua proveniente del suelo fértil en los estanques con fondo de tierra, lo que además de favorecer el crecimiento de los peces disminuye el costo de fertilización de los estanques ${ }^{6,11}$.

Ejemplares de Pseudoplatystoma corruscans en tanques-red alcanzaron valores inferiores en crecimiento, peso y longitud al ser comparados con aquellos criados en estanques de tierra ${ }^{20}$. Del mismo modo, sábalos criados en tanques-red no obtuvieron buenas ganancias de peso y presentaron un desempeño inferior que aquéllos mantenidos en estanques durante todo el experimento ${ }^{10}$, quizás por tratarse de una especie iliófaga que se alimenta del fondo de los estanques ${ }^{15}$. Los resultados aquí obtenidos concuerdan con las consideraciones postuladas por investigadores brasileños que recomiendan considerar las condiciones fisiológicas en situaciones naturales, para que los animales no sean sometidos a condiciones desfavorables con perjuicio del desempeño y producción ${ }^{23}$. Sin embargo, otros no encontraron diferencias en relación al peso medio/ pescado al cultivar Brycon amazonicus en tanques-red y estanques de tierra ${ }^{2}$.

En otros ensayos se mantuvieron sábalos adultos en acuarios con fondo arenoso y abundantes plantas acuáticas durante casi un año, observando que los mismos no se alimentaban y se fueron convirtiendo paulatinamente en "formas de inanición" ". En contraste, peces de gran porte se lograron mantener durante 18 meses en piletas previamente acondicionadas para formar una capa de detritos suficiente como para satisfacer las necesidades de los peces ${ }^{24}$. Sin embargo, otros no encontraron diferencias en relación al peso medio/pescado, al cultivar Brycon amazonicus en tanques-red y estanques de tierra ${ }^{2}$. Cultivando $P$. lineatus a una densidad de 3,2 peces $/ \mathrm{m}^{2}$ en estanques de concreto cuyo fondo fue abonado con abundante material orgánico, sin suministrar alimento balanceado, se obtuvo un crecimiento satisfactorio pero inferior a otras experiencias ${ }^{16}$.

En el presente estudio, si bien se observó que el crecimiento en estanques con fondo de tierra fue superior al obtenido en 
tanques australianos con fondo de cemento, la correcta fertilización y el mantenimiento de la calidad del agua en estos últimos permitió que los sábalos se desarrollen con buenos niveles de supervivencia, indicando que la producción de esta especie es posible en tales condiciones, pero con ganancia de peso más baja. Así, el suministro de una mayor cantidad de materiales orgánicos tanto como el agregado de fertilización inorgánica que aporte diferentes minerales al sistema, podría permitir que los sábalos mejoraran su crecimiento alcanzando guarismos similares a los estimados en estanques construidos con fondo de tierra.

En este contexto, el análisis del zooplancton presente en los estanques así como su evolución durante los cultivos, podría resultar determinante a la hora de explicar la ganancia de peso de los sábalos, ya que este componente del ecosistema del estanque contiene la mayoría de las sustancias nutritivas requeridas por los peces, especialmente aminoácidos y ácidos grasos esenciales, siendo además fuente importante de vitaminas y minerales ${ }^{13,17,21,25}$.

En conclusión, los resultados obtenidos demuestran que los estanques con fondo de cemento no permiten que el sábalo se desarrolle como lo hace en estanques con fondo de tierra. Sin embargo, representan una alternativa para el cultivo de la especie, que debería continuar evaluándose con el objeto de lograr el mantenimiento de una adecuada cantidad de material orgánico que satisfaga las necesidades de los peces.

\section{REFERENCIAS}

1. Angelescu V, Gneri FS. 1949. Adaptaciones del aparato digestivo al régimen alimentario en algunos peces iliófagos del Río Uruguay y Río de la Plata. Rev Inst Nac lnv Cienc Nat. 1: 161-272.

2. Arbeláez GA, Fracalossi DM, Fim JD. 2002. Composição corporal de tambaqui, Colossoma macropomum, e matrinxã, Brycon cephalus, em sistemas de cultivo intensivo, em Igarapé, e semi-intensivo, em Viveiros. $R$ Bras Zootec 31: 1059-1069.

3. Bonetto AA, Castello HP. 1985. Pesca y Piscicultura en aguas continentales de América Latina, Publ Secr Gral OEA, Ser Biol No 31,Washington, USA, 118 p.

4. Britsky HA. 1972. Peixes de água doce de Estado de São Paulo. Sistemática. In: Poluição e piscicultura (Ed.Comissão Interestadual Bacia Paraná-Uruguai), Fac Saúde Públ USP, São Paulo, p. 79-108.

5. Camargo MP, Martínez CB. 2006. Biochemical and physiological biomarkers in Prochilodus lineatus submitted to in situ tests in an urban stream in southern Brazil. Environ Toxicol Pharmacol 21: 61-69.

6. Corral ML, Grizel H, Montes J, Polanco E. 2000. $\mathrm{La}$ acuicultura: biología, regulación, fomento, nuevas tendencias y estrategia comercial. Tomo I: Análisis del desarrollo de los cultivos: medio, agua y especies. Edit. Fund. Alfonso Martín Escudero, Madrid, 239 p.

7. Escobar E, Regidor HA, Iwaszkiw J, Mosa SG. 2011. Análisis comparativo de la fecundidad del sábalo Prochi- lodus lineatus en ambientes lóticos y lénticos de la Argentina. Aquatic 34: 1-11.

8. Espinach A, Sánchez RP. 2007. Proyecto evaluación del recurso sábalo en el Paraná. Informe de los resultados de la primera etapa (2005-2006) y medidas de manejo recomendadas. Publ. Serie Pesca y Acuicultura: estudios e investigaciones aplicadas, Secret. Agric. Ganad. Pesca y Alim., Buenos Aires, 80 p.

9. Furuya VR, Hayashi C, Furuya WM, Soares CM, Galdioli EM. 1999. Influência de plâncton, dieta artificial e sua combinação, sobre o crescimento e sobrevivência de larvas de curimbatá (Prochilodus lineatus). Acta Scientiarum 21: 699-703.

10. Hainfellner P. 2011. Desenvolvimento ovariano do Prochilodus lineatus em dois sistemas de produção: tanques rede e viveiros escavados, Publ. Univ. Estad. Paulista Centro Aquic., Jaboticabal (São Paulo), 73 p.

11. Hickling CF. 1974. Recommendations for constructions and management of brackishwater aquaculture ponds in areas with acid sulfate soils. Circular FAO Fisheries 658: 243-260.

12. InfoStat. 2002. InfoStat versión 1.1. Grupo InfoStat, FCA, Universidad Nacional de Córdoba, Argentina.

13. Kubitza F. 1998. Nutrição e alimentação dos peixes cultivados, Ed. Jundiaí, São Paulo (Brasil), 123 p.

14. Ministerio de Agricultura, Ganadería y Pesca (Argentina). 2011. Productos de río. 2011. http://www.minagri.gob. ar/site/pesca/pesca_continental/05-estad\%C3\%ADsticas / archivos/010000_Productos\%20de\%20r\%C3\%ADo.php.

15. Moraes MF, Barbola IF, Guedes EA. 1997. Alimentação e relações morfológicas com o aparelho digestivo do “curimbatá" Prochilodus lineatus (Valenciennes) (Osteichthyes, Prochilodontidae), de uma lagoa do sul do Brasil. Rev Bras Zool 14: 169-180.

16. Parma MJ. 1992. Comportamiento y crecimiento de Prochilodus lineatus (Pisces, Curimatidae) en condiciones controladas. Rev Asoc Cienc Nat Litoral 23: 9-20.

17. Pelli A, Dumont R, Silva J, Gonçalves S, Souza D, Barbosa N. 1996. Início de ingestão de ração por pacú (Piaractus mesopotamicus Holmberg, 1887), curimba (Prochilodus scrofa Steinchdacner, 1881) e piau (Leporinus friderici Bloch, 1794) em condições de criação semi-intensiva. Memorias de Simpósio Brasileiro de Aqüicultura, Sete Lagoas (Brasil), 88 p.

18. Prieto MJ, Rosa PV, Ferreira G, Araújo F. 2006. Tipo de alimento, sobrevivência e desempenho inicial de pós-larvas de pacu (Piaractus mesopotamicus). Ciênc e Agrotec 30: 1002-1007.

19. Reidel A, Oliveira LG, Piana PA, Lemainski D, Bombardelli RA, Boscolo WR. 2004. Avaliação de rendimento e características morfométricas do curimbatá Prochilodus lineatus (Valenciennes, 1836) e do piavuçu Leporinus macrocephalus (Garavello \& Britski, 1988) machos e fêmeas. Revista Varia Scientia 4: 71-78.

20. Scorvo JD, Romagosa E, Ayroza LM, Frascá CM. 2008. Desempenho produtivo do pintado, Pseudoplatystoma corruscans (Spix \& Agassiz, 1829), submetidos a diferentes densidades de estocagemem dois sistemas de criação: intensivo e semi-intensivo Inst Pesca São Paulo 34: 181-188. 
21. Sipaúba LH, Rocha O. 2003. Produção de plâncton (fitoplâncton e zooplâncton) para alimentação de organismos aquáticos, Ed. RIMA (São Carlos, Brasil), 106 p.

22. Sverlij SB, Espinach A, Orti G. 1993. Sinopsis de los datos biológicos y pesqueros del sábalo, Prochilodus $l i$ neatus (Valenciennes, 1847). In: Sinopsis sobre la Pesca $\mathrm{N}^{\circ}$ 154 (FAO Ed.), Roma, 64 p.

23. Urbinati EC, Carneiro PC. 2004. Práticas de manejo e estresse dos peixe em piscicultura. In: Tópicos especiais em piscicultura de água doce tropical intensiva (Cyrino J, Urbinati E, Fracalossi D, Castagnolli N, eds.), Editorial Tecart, São Paulo; p 171-193.
24. Vidal JC. 1967. Contribución al estudio biológico del sábalo de los ríos Paraná y Uruguay. Publ. Secr. Est. Agric.y Ganad. (Buenos Aires), p. 1-51.

25. Zimmermann S, Jost HC. 1998. Recentes avanços na nutrição de peixes: a nutrição por fases em piscicultura intensiva. Memorias Simpósio sobre manejo e Nutrição de Peixes, Piracicaba- SP (Brasil), p. 123-162.

\section{Revista Veterinaria obtuvo el máximo nivel de categorización del CAICYT-CONICET}

Tras el pertinente proceso de evaluación según criterios de calidad editorial, en setiembre de 2005 CAICYT-CONICET ha clasificado a nuestra publicación con Categoría 1 (nivel superior de excelencia), con lo cual pasa a integrar el Catálogo Latindex (folio 14022). La Dirección de Revista veterinaria agradece a quienes colaboraron para obtener tan importante distinción. Ver: http://www.latindex. unam.mx/busquedas/catalogotitulo.html 